\title{
Ionic Lanthanum Passage Across Cerebral Endothelium Exposed to Hyperosmotic Arabinose
}

\author{
K. Dorovini-Zis ${ }^{2}$, M. Sato ${ }^{3}$, G. Goping ${ }^{1}$, S. Rapoport ${ }^{4}$, and M. Brightman ${ }^{1}$ \\ 1 Laboratory of Neuropathology and Neuroanatomical Sciences, NINCDS National Institutes of Health, Bethesda, MD 20205, USA \\ 2 Dept. of Pathology, University of Michigan Medical School, Ann Arbor, MI 48109, USA \\ 3 Health Administration Center, Kyoto University, Kyoto, Japan \\ 4 Laboratory of Neurosciences, National Institute of Aging, Baltimore, MD 21224, USA
}

Summary. Hyperosmotic media infused into the cerebral circulation open the blood-brain barrier to protein and colloid. The mechanism whereby such substances cross the affected vessels is still disputed. We describe here the transendothelial route taken by ionic lanthanum $\left(\mathrm{La}^{3+}\right)$, a small electron-dense tracer which, unlike colloidal lanthanum, can be administered to the living animal. In adult rats, $2.9 \mathrm{ml}$ of hyperosmotic $(1.4 \mathrm{M})$ arabinose was infused into the internal carotid artery as a $30-\mathrm{s}$ bolus, followed by $5 \mathrm{mM} \mathrm{LaCl}_{3}$. To find the extravasated $\mathrm{La}^{3+}$, which is invisible by light microscopy, horseradish peroxidase (HRP) was injected simultaneously into the femoral vein. The hyperosmotic treatment resulted in exudation of both HRP and $\mathrm{La}^{3+}$ primarily around cerebral arterioles. The $\mathrm{La}^{3+}$ crossed arterioles through successive tight junctions between endothelial cells. Although the tight junctions were not discernibly opened, they must have become permeable because the extracellular pools between successive tight junctions were penetrated by the $\mathrm{La}^{3+}$. These pools are normally inaccessible to $\mathrm{La}^{3+}$. Luminal and abluminal pits and cytoplasmic vesicles, some of them containing $\mathrm{La}^{3+}$, formed intraendothelial clusters. Their role, if any, in the transfer of ion remains uncertain.

Key words: Blood-brain barrier - Ionic lanthanum Tight junctions

\section{Introduction}

The mechanisms whereby cerebral blood vessels exposed to hyperosmotic solutions allow macromolecules to pass from blood to brain have yet to be fully

Offprint requests to: Milton W. Brightman, $\mathrm{PhD}, \mathrm{Head}$, Section of Neurocytology, NINCDS, Bldg. 36, Rm. 3B26, National Institutes of Health, Bethesda, MD 20205, USA established. The hypothesis that such solutions shrink the endothelium and thus open the barrier (Rapoport et al. 1972; Rapoport 1978) has received morphological support by the penetration of horseradish peroxidase (HRP) into extracellular pools between successive tight junctions that normally make such pools inaccessible to the protein (Brightman et al. 1973). The entry of a smaller molecule, colloidal lanthanum, into interjunctional pools, has been also regarded to result from junction patency. The lanthanum was injected into the cerebral vasculature after fixation of the vessels exposed to a hyperosmotic solution (Nagy et al. 1979).

An alternative explanation of how HRP escapes from cerebral vessels infused with hyperosmotic solutions is the formation of vesicles by invaginations of the luminal plasma membrane. The vesicles then are considered to transfer plasma and its contents to the opposite, abluminal face of the endothelium (Westergaard 1980). Tracers rarely, if ever, penetrated tight junctions in such vessels (Westergaard 1980). Hyperglycemia, another condition that increases barrier permeability, is likewise unaccompanied by morphological changes in the component strands of frozencleaved tight junctions (Shivers 1979). These observations have been taken to reinforce the conclusion that the increase in vessel permeability during hyperosmotic infusion is due to an active, transendothelial, vesicular transfer rather than to a passive, intercellular diffusion.

In the experiments reported here, the smallest, electron-dense tracer available, ionic lanthanum $\left(\mathrm{La}^{3+}\right)$, has been used. $\mathrm{La}^{3+}$ passage, therefore, should be more closely comparable to that of plasma ions than is HRP. Colloidal lanthanum which clots blood rapidly, is administered by perfusing it, together with fixative, after first flushing out the blood with fixative alone. In contrast, small concentrations of ionic lanthanum can be infused i.v. in living rodents without apparent ill effects and the probe ion can be subsequently detected with the electron microscope. 
The intention of the present experiment was to see whether ionic lanthanum traverses the cerebral endothelium by the same route taken by the much larger substance, the glycoprotein, horseradish peroxidase (MW 40,000), following the intracarotid infusion of hyperosmotic arabinose.

\section{Material and Methods}

Inasmuch as lanthanum is indiscernible by light microscopy and the affected segments of blood vessels are randomly and unpredictably distributed, it was necessary to infuse HRP, detectable by light microscopy, at about the same time as lanthanum. The reaction product of HRP would be expected to mark at least some of the sites of $\mathrm{La}^{3+}$ exudation. A hyperosmotic solution of arabinose was used to open the blood-brain barrier. The threshold concentration of arabinose at which exudates of Evans blue-albumin are consistently formed, is 1.4 M (Chiueh et al. 1978). The osmolarity of all solutions was determined initially with an osmometer.

Adult male Osborne-Mendel rats, weighing $260-350 \mathrm{~g}$, were anesthesized by i.p. injection of sodium pentobarbital $(50 \mathrm{mg} / \mathrm{kg})$. A polyethelene catheter (PE 50) was tied into the right external carotid artery for retrograde infusion of arabinose and $\mathrm{LaCl}_{3}$. Hypertonic fluid, infused into the external carotid vessel, was immediately swept into the internal carotid and then the ipsilateral cerebral hemisphere by systemic blood pressure. Cerebral circulation was re-established immediately after infusion ceased.

Three groups of animals were treated as follows:

\section{Group $I$}

A hypertonic solution of (1.4 M) L(t)-arabinose (Sigma Chemical, St. Louis, MO, USA) in $0.9 \%(\mathrm{w} / \mathrm{v}) \mathrm{NaCl}$ was infused in a vol. of $2.9 \mathrm{ml}$ over a period of $30 \mathrm{~s}$ with a constant flow pump. About $15-20 \mathrm{~s}$ later, $1 \mathrm{ml}$ of $0.9 \% \mathrm{NaCl}$ containing $3-5 \mathrm{mM} \mathrm{LaCl}_{3}$ was infused over a period of $3 \mathrm{~min}$. HRP, (Sigma, Type VI), $0.25 \mathrm{mg} / \mathrm{g} \mathrm{b.w.,} \mathrm{dissolved} \mathrm{in}$ $0.6 \mathrm{ml}$ of $0.9 \% \mathrm{NaCl}$, was injected in to the left femoral vein $30 \mathrm{~s}$ after the $\mathrm{LaCl}_{3}$ injection.

\section{Group II}

HRP, $(0.6 \mathrm{ml})$ was infused into the left femoral vein. Hypertonic arabinose was injected into the carotid circulation 2-3 min after HRP injection. $\mathrm{LaCl}_{3}(5 \mathrm{mM})$ was infused $3 \mathrm{~min}$ following the arabinose.

\section{Group III (Control Animals)}

Isotonic arabinose solution was administered through the catheterized right external carotid artery in a vol. of $2.9 \mathrm{ml}$ over a 30 -s period. $\mathrm{LaCl}_{3}(5 \mathrm{mM})$ was injected $1 \mathrm{~min}$ later. HRP was infused into the left femoral vein 1-2 min after lanthanum injection. The vol. of $\mathrm{LaCl}_{3}$ and HRP were the same as in the experimental groups. The circulation times allotted for the solutions are presented in Table 1.

The brains of all three groups of animals were fixed by perfusion with aldehydes, containing $5 \mathrm{mM} \mathrm{LaCl}_{3}, 1-4 \mathrm{~min}$ following the last injection. The initial fixative contained $1.25 \%$ glutaraldehyde and $1 \%$ paraformaldehyde in $0.1 \mathrm{M}$ cacodylate buffer $(\mathrm{pH} 7.35)$. The perfusion was continued with the more concentrated fixative containing $5 \%$ glutaraldehyde and $4 \%$ paraformaldehyde in $0.1 \mathrm{M}$ cacodylate buffer. The brains were removed $2 \mathrm{~h}$ after fixation and stored in sodium cacodylate buffer, containing $5 \mathrm{mM} \mathrm{LaCl}_{3}$, overnight at
Table 1. Circulation time alloted for arabinose and tracers including, the time taken for infusion plus circulation

\begin{tabular}{llll}
\hline Groups & \multicolumn{1}{c}{ Minutes } & & \\
\cline { 2 - 3 } & & HRP & $\mathrm{La}^{3+}$ \\
\hline Hyperosmotic & arabinose & & \\
I & $6.5-9.5$ & $2-5$ & $5.5-8.5$ \\
II & $4.5-7.5$ & $8.5-11.5$ & $4-7$ \\
Isosmotic arabinose & & \\
III & & \\
(Control) & $8.5-11.5$ & $2-5$ & $7-9$ \\
\hline
\end{tabular}

$4{ }^{\circ} \mathrm{C}$. Sections, $50 \mu \mathrm{m}$ thick, were cut with a Smith-Farquhar chopper. Every tenth section was examined by light microscopy. All tissue slices were incubated in 3,3'-diaminobenzidine for $1 \mathrm{~h}$ at about $5-10^{\circ} \mathrm{C}$, for the histochemical demonstration of the HRP. Regions with the exudates were excised from the chopped sections, then postfixed in $2 \%$ buffered solution of osmium tetroxide. Some pieces were then mordanted en bloc with uranyl acetate. Thin plastic sections from both mordanted and unmordanted slices were examined electron microscopically without heavy metal staining.

\section{Results \\ Controls}

In the control animals that had been infused with isosmotic arabinose, HRP reaction product stained intensely the circumventricular regions that are normally devoid of a blood-brain barrier. A few, faint deposits of HRP infiltrated, segmentally, the wall of very few vessels at or near the cortical surface.

Lanthanum deposits could be visualized only by electron microscopy. In control animals, $\mathrm{La}^{3+}$ was retained within the lumen of blood vessels and usually stuck in patches to the luminal surface of the endothelium. A few luminal pits, cytoplasmic vesicles (Figs. 1C, 5B), and multivesicular bodies contained the tracer, although some of the pits may have been washed free of the $\mathrm{La}^{3+}$ during the aldehyde perfusion. The lanthanum was deposited either as an amorphous mass (Figs. 1,8), needle-like crystals, (Fig. 3A-C) or as very small, electron-dense granules (Figs. 3B, 4D). Endothelial tight junctions were not usually permeated by the ionic lanthanum, which usually stopped at the first, luminal junction (Fig. 1). Occasionally, however, $\mathrm{La}^{3+}$ was able to enter the intercellular pool beyond the first junction; the remaining pools were invariably free of deposit (Fig. 1 A, B). In such sections (Fig. 1C), the interjunctional pools could be distinguished from confluent vesicles because the contour of the pools was ovoid (Fig. 2), whereas that of single or intercommunicating vesicles was round (Figs. 5, 6, 8). HRP was 

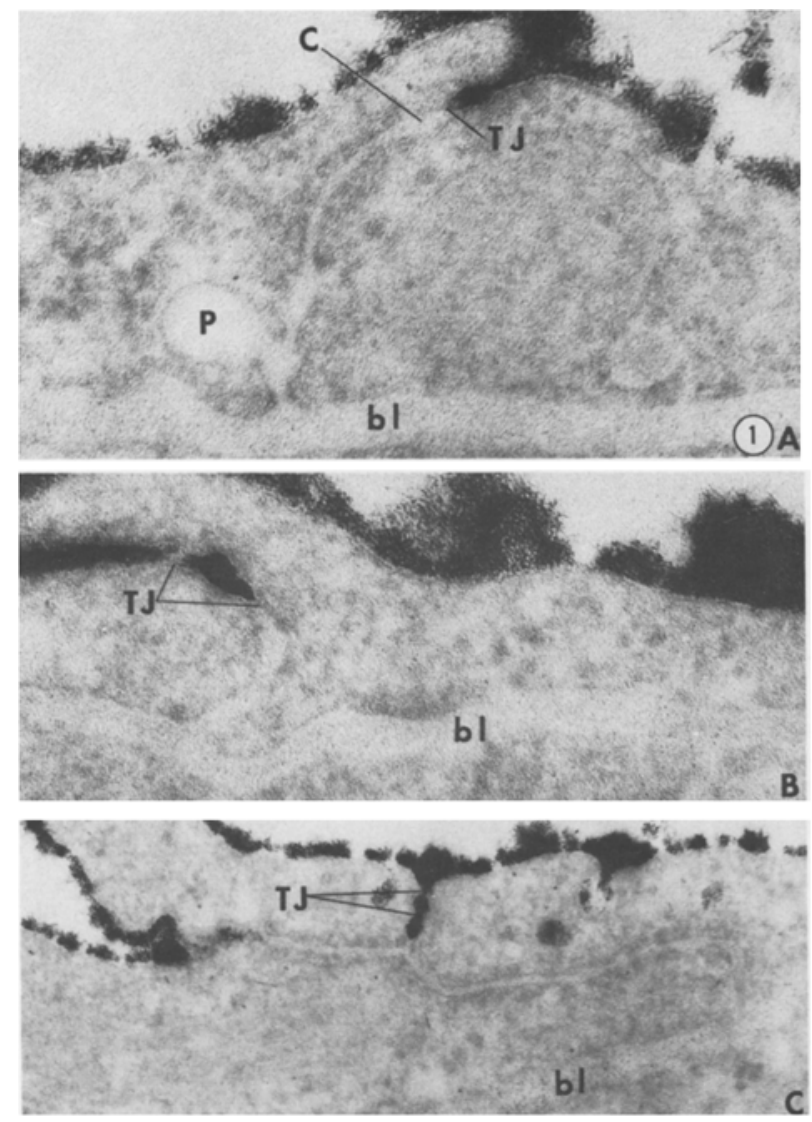

Fig. 1A-C. Variations in controls: isosmotic arabinose, $\mathbf{A ~ L a}^{\mathbf{3 +}}$ coats luminal surface of endothelium passes into cleft $(C)$ between two endothelial cells and stops at a luminal tight junction $(T J)$. Distended pit $(P)$ communicates with cleft. Pit, cleft, and basal lamina $(b l)$ are all free of $\mathrm{La}^{3+}$. Control. $\times 97,200 . \mathrm{B} \mathrm{La}^{3+}$ appears to have passed through one TJ and stops at a second one leaving cleft and basal lamina $(b l)$ free of deposit. Control. $\times 97,200 . \mathbf{C ~ L a}^{3+}$ has passed two TJs. Although the remainder of clefts are free of $\mathrm{La}^{3+}$ there appears to be, atypically, a fine, granular deposit in the basal lamina $(b l)$. An alternative, though less likely interpretation, is that two labeled vesicles have become confluent. If so, the abluminal one may not communicate with the basal lamina, because no "puff" of $\mathrm{La}^{3+}$ has entered the lamina from this vesicle. Control. $\times 60,170$

similarly distributed. Ionic lanthanum, whether amorphous, granular or needle-shaped, did not enter any perivascular basal lamina.

The distribution of lanthanum in the choroid plexus indicated whether the lanthanum was in the colloidal or ionic state, because colloidal lanthanum cannot penetrate the tight junctions between the epithelial cells (Brightman and Reese 1969) whereas ionic lanthanum can (Castel et al. 1974). In our experiments, $\mathrm{La}^{3+}$ left the choroidal vessels and in. a few instances, passed through the epithelial tight junctions laying next to the ventricular lumen. It could not be ascertained with certainty, however, whether or not the most luminal junction had also been penetrated because no distinct,

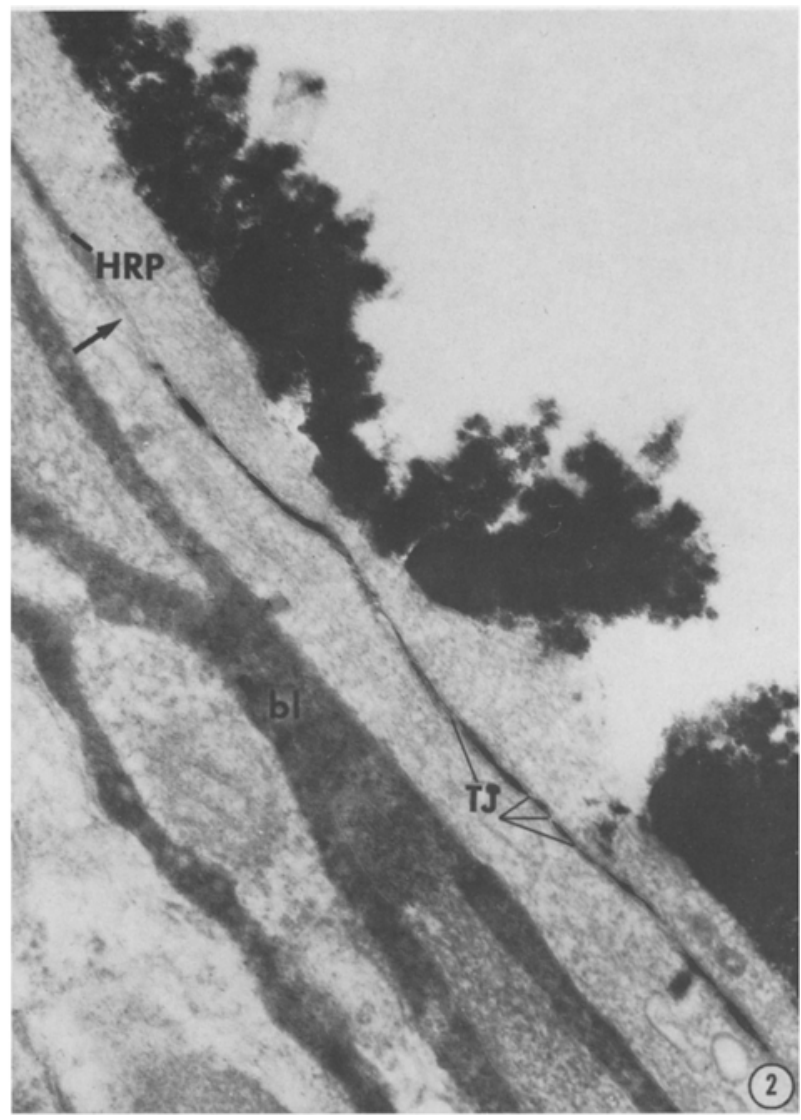

Figs. 2-4. Infusion of $1.4 \mathrm{M}$ arabinose

Fig. 2. $\mathrm{La}^{3+}$ has penetrated endothelial cleft past at least four tight junctions $(T J)$ but stops abruptly to leave a pool (arrow) free of both $\mathrm{La}^{3+}$ and peroxidase $(H R P)$, in this arteriole. The HRP front has moved retrogradely into cleft. Basal lamina $(b l)$ appears to contain both $\mathrm{La}^{3+}$ granules and HRP. $\times 50,545$

continuous deposit could be detected on the free surface of the epithelium. Cytoplasmic vesicles within the basal regions of the epithelial cells contained $\mathrm{La}^{3+}$ while those in the apical regions did not.

\section{Hyperosmotic Treatment}

In those rats that had received hyperosmotic arabinose, numerous, punctate, exudates - round or oval in shape - surrounded portions of blood vessels in the infused hemisphere. The contralateral hemisphere also contained some spots of extravasation but they were fewer and variable in number. On the infused side, exudates usually appeared in the outer layers of the cortices, varied in size and, within a few minutes, had become confluent with their neighbors. Both pial and large, penetrating vessels were so affected. Arterioles were, by far, the most common type of blood vessel near the center of an extravasation, although venules and capillaries were also involved. Temporal and parietal 

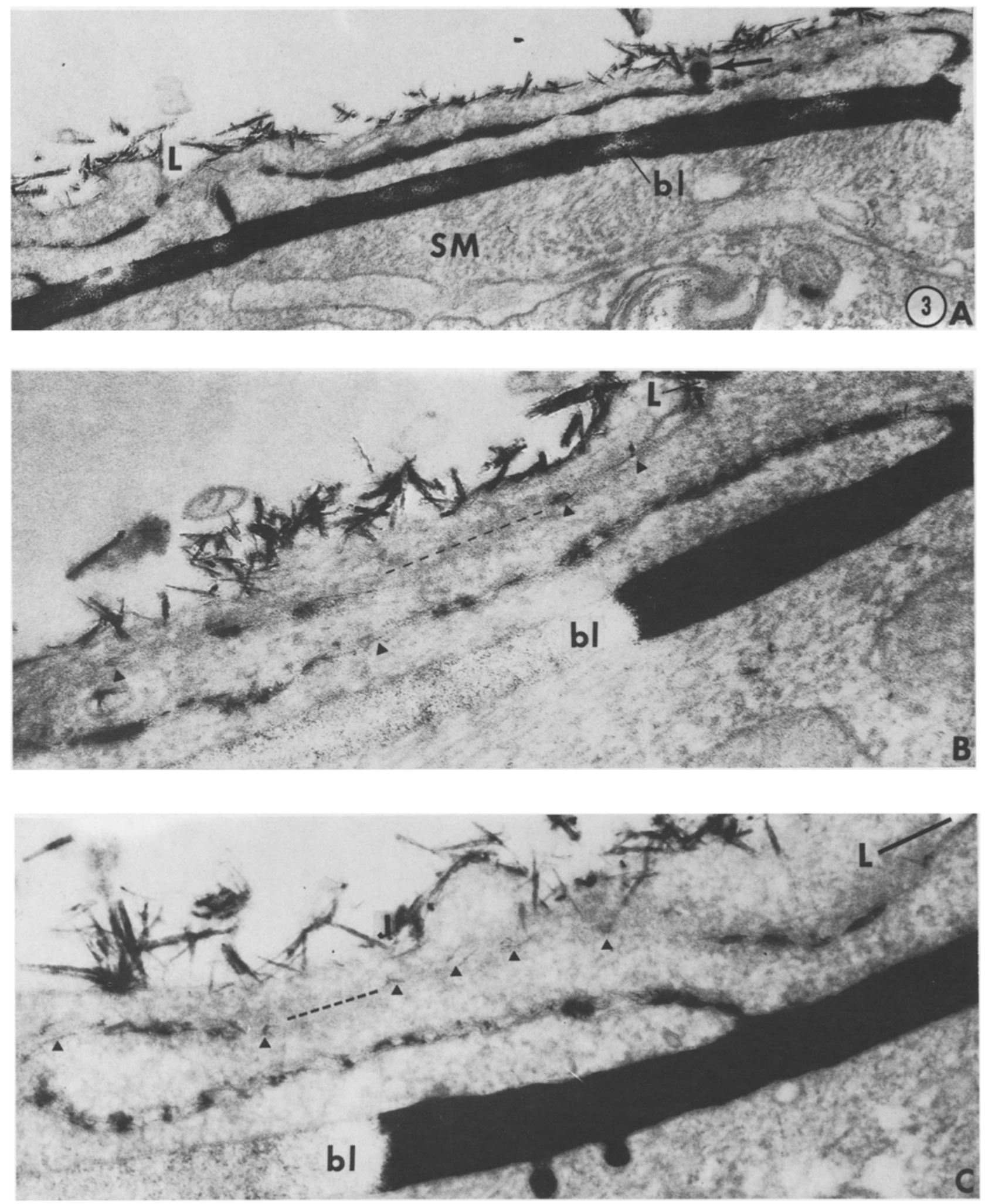

Fig. 3 A-C. Interendothelial clefts contain $\mathrm{La}^{3+}$ extending from their luminal $(L)$ end to basal lamina $(b l)$. Small but significant deposits are marked by arrow heads. A Pit (arrow) containing $\mathrm{La}^{3+}$ is confluent with cleft. Smooth muscle cell $(S M) . \times 100,000$. B, C Sequential sections with $\mathrm{La}^{3+}$ in cleft, but at different regions. Absence (dashed line) of deposits from a region in one section are present in that region in the sequential section. In basal lamina $(b l)$, a continuous column of $\mathrm{La}^{3+}$ ends abruptly near region of fine granules. Two sarcolemmal pits are labeled at the bottom of C. B, C: $\times 104,000$ 

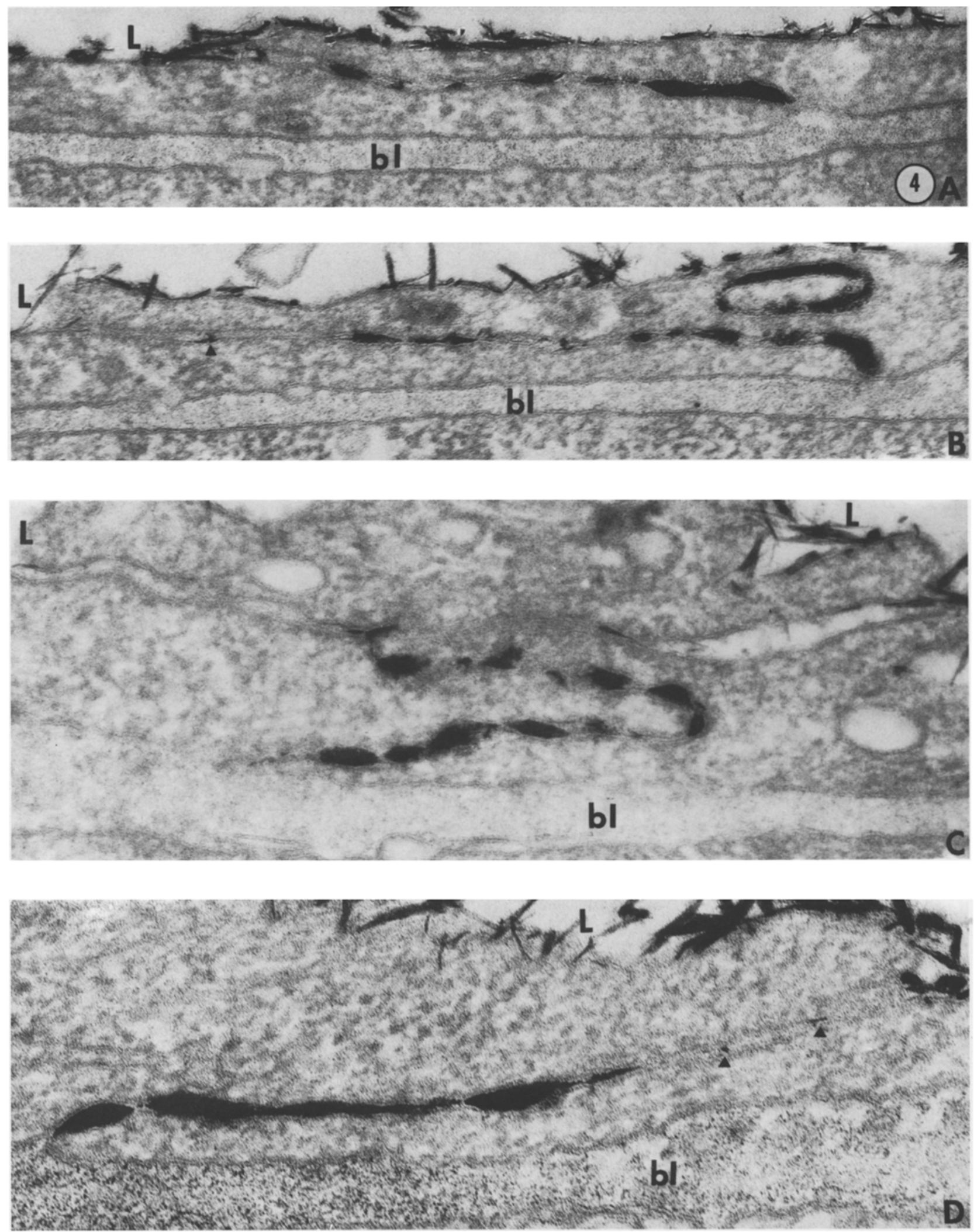

Fig. 4 A-D. $\mathrm{La}^{3+}$ throughout cleft, from its luminal end $(L)$ to basal lamina $(b l)$. However, in $\mathbf{A}$ and $\mathbf{B}$, only sparse grains $\left(\mathrm{La}^{3+}\right.$ ?) in basal lamina $(b l)$ at this level. D Luminal parts of cleft are narrow but patent with small amount of La ${ }^{3+}$ (arrow heads). Substantial granular deposit in basal lamina $(b l) . \mathbf{A}: \times 104,000 ; \mathbf{B}: \times 104,000 ; \mathbf{C}: \times 192,000 ; \mathbf{D}: \times 156,000$ 

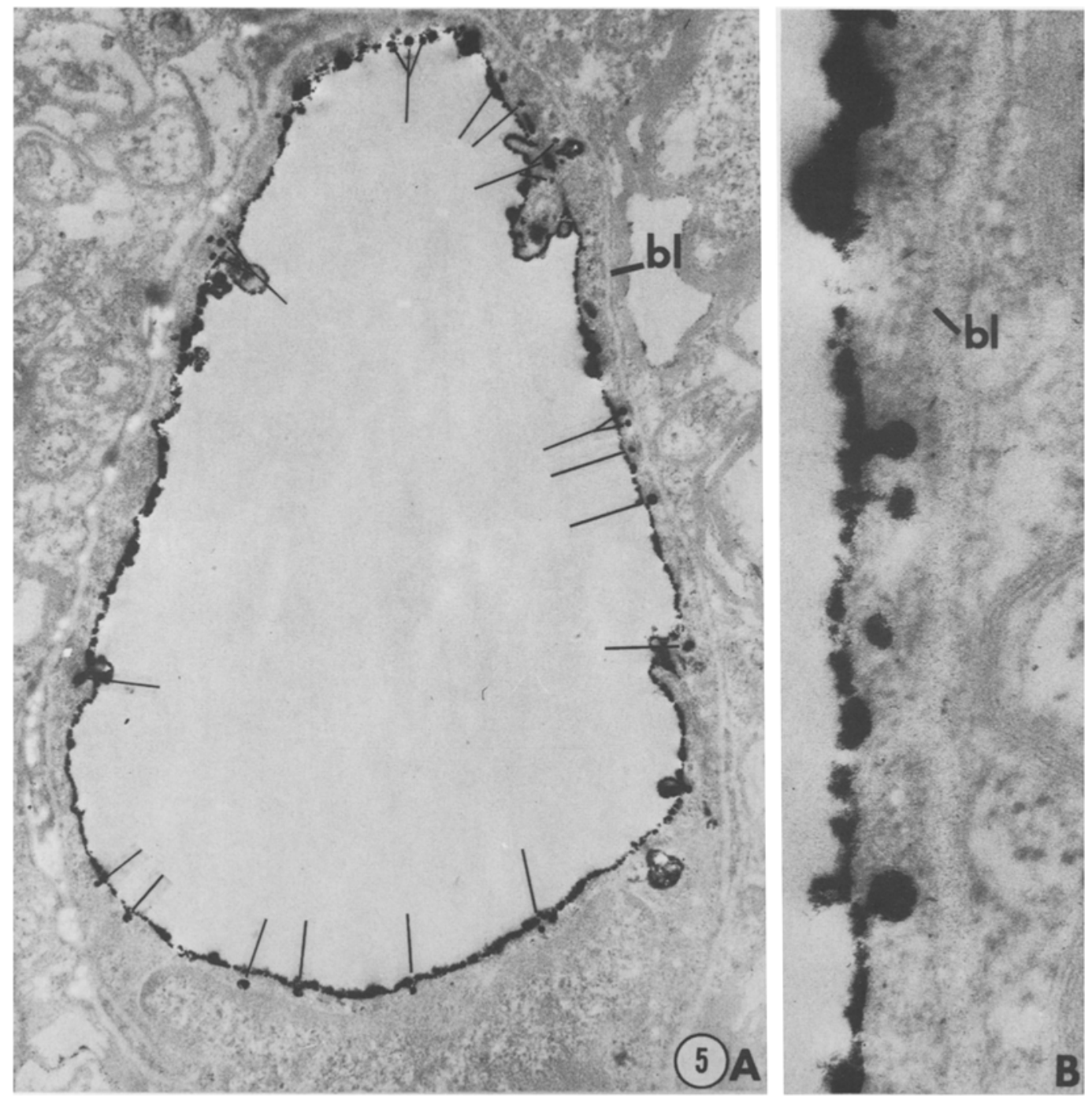

Fig. 5 A, B. Isosmotic arabinose controls. $\mathrm{A} \mathrm{La}^{3+}$ in pits and vesicles (lines) of endothelium. No $\mathrm{La}^{3+}$ in basal lamina ( $b l$ ) or neuropil clefts. $\times 17,000$. B Luminal pits contain $\mathrm{La}^{3+}$. No abluminal pits in these particular specimens. No $\mathrm{La}^{3+}$ in basal lamina $(b l) . \times 78,000$

regions of the cerebral cortex and the brain stem contained the greatest number of exudates. There was no difference in the number, distribution, density of brown color, or degree of confluence of the exudates between either of the two experimental groups receiving the hyperosmotic arabinose. The neural parenchyma around the affected vessels was permeated by the HRP so that cells and their processes were sharply outlined by reaction product. As in the controls, only the walls of some vessels were infiltrated with HRP while the surrounding neuropil was free of the protein deposit.

Both HRP and $\mathrm{La}^{3+}$ escaped from the same blood vessel, but occasionally, only one of them left the vessel lumen. The tracers were distinguishable primarily because of the greater electron density of the lanthanum. Where the lanthanum formed a finely granular ag- gregate it could be partially or totally obscured by the reaction product of HRP. In some of the clefts between endothelial cells, a column of lanthanum extending from the vessel lumen faced a column of HRP reaction product extending into the same cleft from the perivascular basal lamina (Fig. 2). Usually, the two fronts were separated by a tight junction or by an empty interjunctional pool. A continuous column of HRP extending from lumen to basal lamina was not encountered in any of the specimens, although it is conceivable that the HRP have been masked by the more electron-dense lanthanum. At some sites where the barrier had been disrupted, both tracers were able to infiltrate the basal lamina and the abluminal pits of the endothelium (Figs. 2,7). The $\mathrm{La}^{3+}$ deposit, even where it was very dense and compact within the basal lamina, 

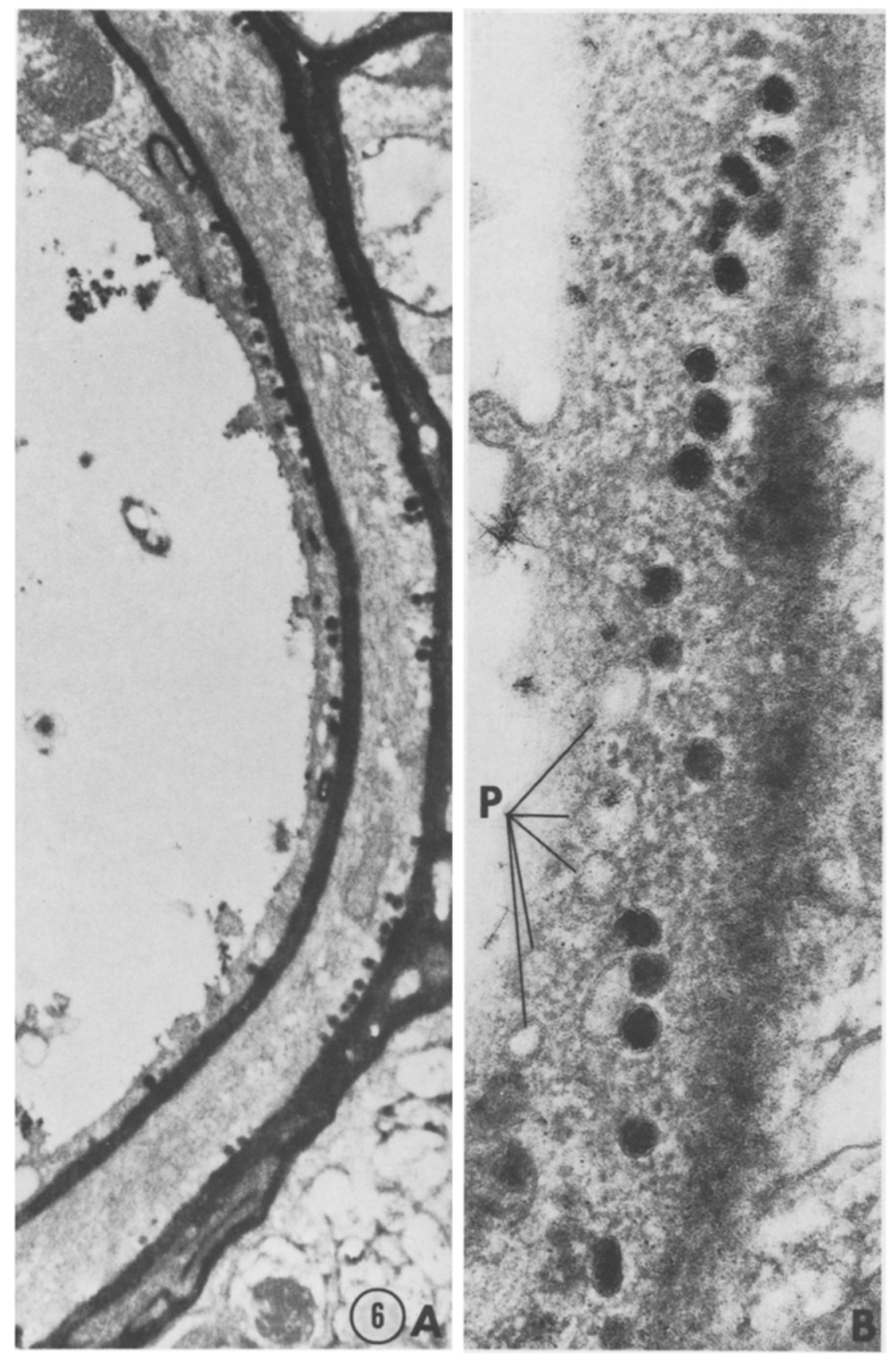

Fig. 6A, B. Almost all labeled pits and vesicles are abluminal $(\mathbf{B})$ and some are clustered. Luminal pits and vesicles $(P)$ do not contain $\mathrm{La}^{3+}(\mathbf{B})$. A arteriole, $\times 24,000$; B capillary, $\times 120,000$

could be abruptly discontinuous; immediately adjacent to a dense deposit, the basal lamina could be either finely stippled by granular aggregates or appear to be devoid of $\mathrm{La}^{3+}$ (Figs. 3B, 4C, 5D).

Thirty-six blood vessels surrounded by exudate were randomly selected and serially sectioned. In 12 of these vessels, there were a total of 15 clefts in which all of the extracellular pools between successive tight junctions had been penetrated by $\mathrm{La}^{3+}$ from luminal to abluminal surfaces (Figs $3 \mathrm{~A}, 5 \mathrm{~A}, \mathrm{C}, \mathrm{D}$ ). Ten of the 12 vessels were arterioles and, in a single section, had only one patent cleft within each vessel. Two of the 12 vessels were arterioles that had two or three patent clefts of a total of four to five clefts per vessel. The remaining 24 vessels did not have clefts that were completely patent but, rather, had only one or two pools that were penetrated by the $\mathrm{La}^{3+}$. Thus, of the arterioles examined, $33 \%$ had at least one open cleft. Where the clefts were patent, the interjunctional lacunae containing $\mathrm{La}^{3+}$ were variably distended. In a single, thin section, the entire series of in tandem lacunae along a cleft could be partially or completely filled with lanthanum. A 


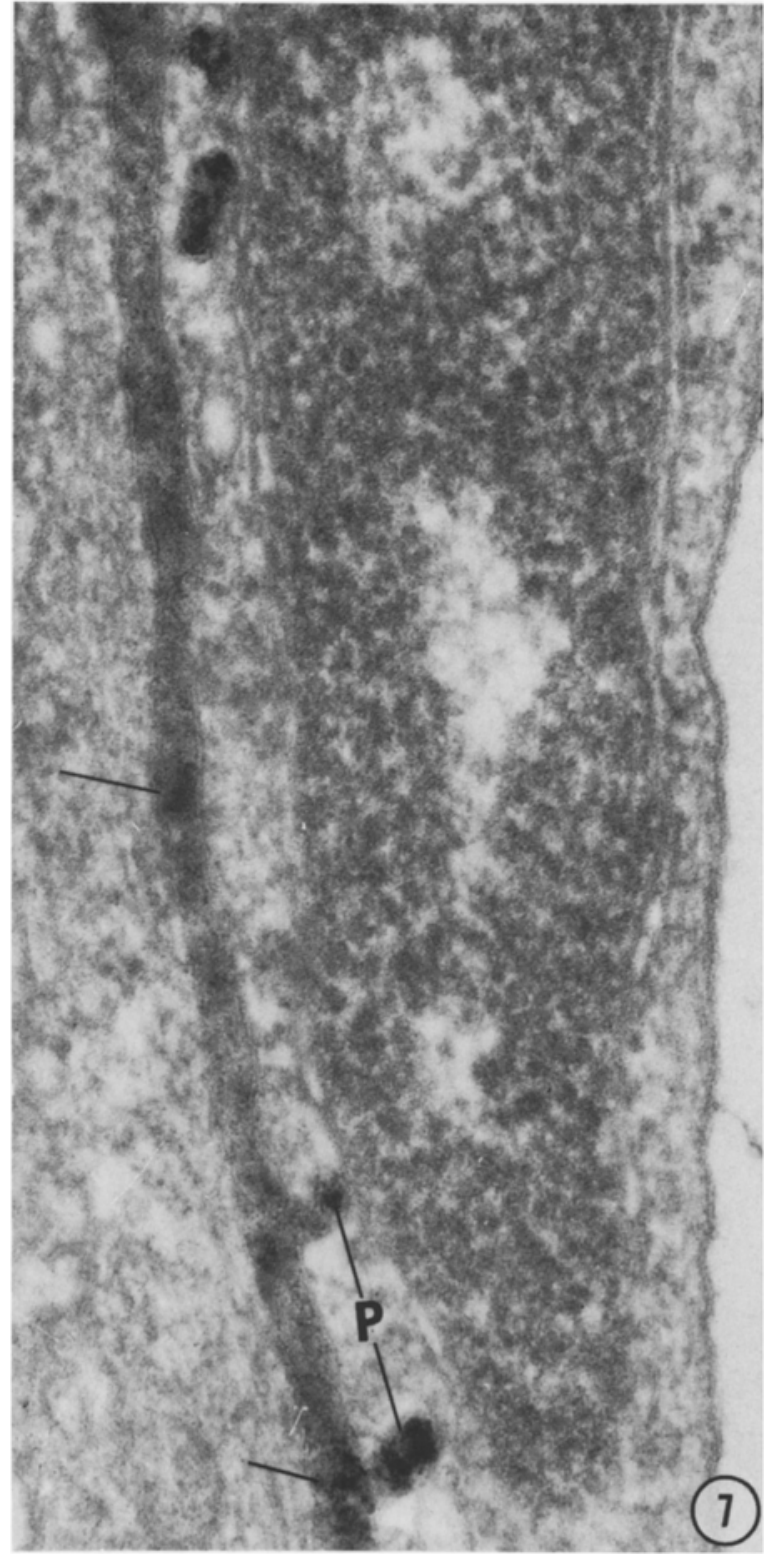

Fig. 7. Focal deposits or "puffs" of $\mathrm{La}^{3+}$ (lines in basal lamina are opposite or near abluminal pits $(P)$ and partly obscured by HRP. $\times 112,000$

column of deposit could thus be traced from luminal to abluminal ends of the cleft in a few such vessels (Figs. 3A, 4A, C, D). Even where the penetration of ionic lanthanum into the cleft was complete and uninterrupted, however, the dense, amorphous, deposit halted at the basal lamina which either appeared empty (Fig. 4B, C) or contained a granular dispersion (Figs. 3B, C; 4A, D). It is emphasized that the entry of $\mathrm{La}^{3+}$ into an entire series of interjunctional pools was found along one cleft in a single section in about one third of the affected vessels. This ratio may be too low
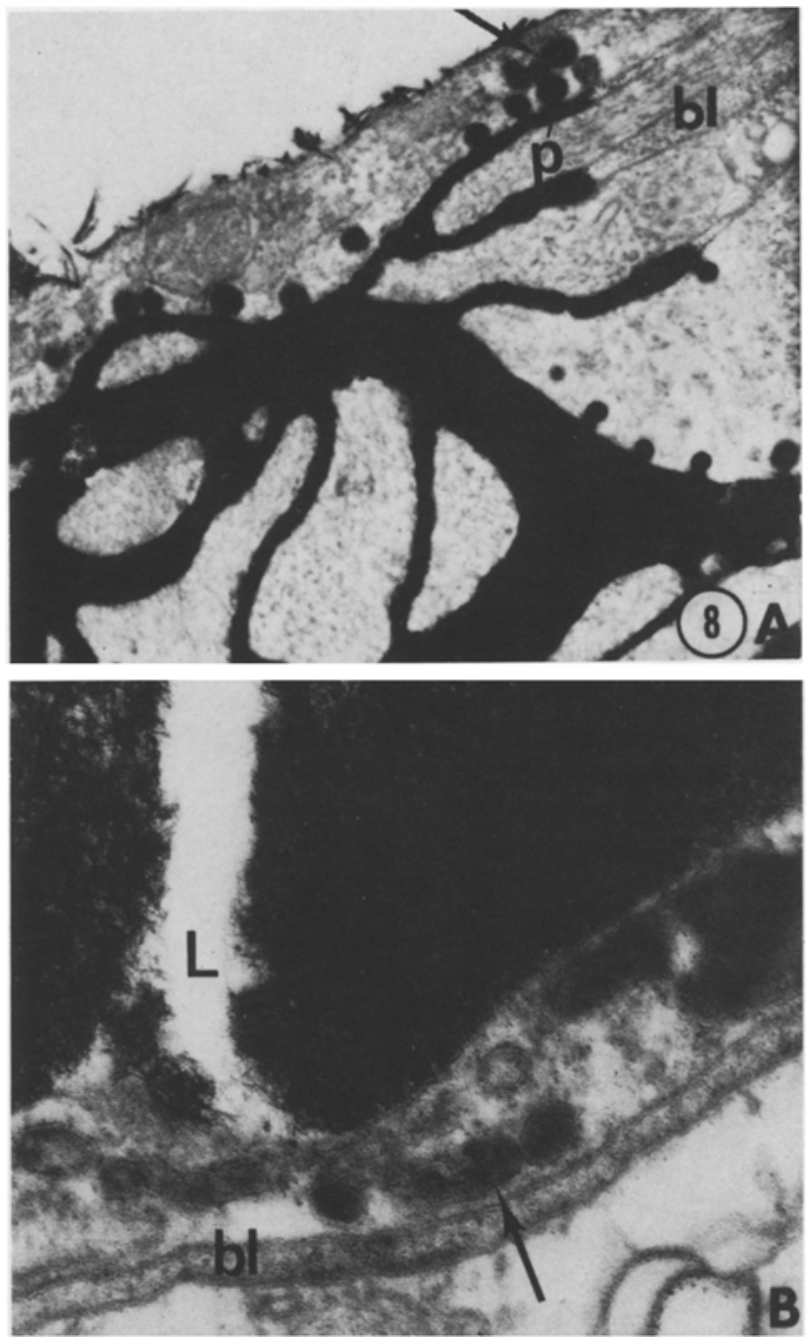

Fig. 8. A Tubule formed by confluent vesicles (arrow) continuous with abluminal pit $(P)$ may have received its $\mathrm{La}^{3+}$ retrogradely from basal lamina $(b l) . \times 48,000$. B Tubule (arrow) within endothelium is labeled primarily with HRP but does not connect vessel lumen $(L)$ with basal lamina $(b l)$ in this section. $\times 110,000$

because most of the clefts undulated out of the plane of section. Thus, the extent and frequency of penetration would be more evident in serial sections.

The number of vesicles and pits - flask-shaped indentations of the luminal and abluminal cell membrane of the endothelium - was augmented in a very few capillaries. Free vesicles within the endothelial cytoplasm were labeled in varying numbers and degree of filling. A number of these vesicles are probably caveolae. Although most vesicles were unlabeled, those that were sometimes formed clusters (Fig. 6A) as they did in controls (Fig. $5 \mathrm{~A}$ ). At some sites, luminal pits and vesicles contained $\mathrm{La}^{3+}$, but the subjacent basal lamina appeared to be completely free of it. Most of the labeled pits were abluminal (Fig. 6A). By about $4.5 \mathrm{~min}$ after 
the hyperosmotic infusion (group II), the shortest interval between injection of arabinose and fixation, neither the pits that were still open to the vessel lumen nor the immediately adjacent, luminal vesicles contained lanthanum. Vesicles that lay deeper in the endothelial cytoplasm did, in contrast, contain lanthanum (Fig. 6B) and a number of them opened onto the labeled basal lamina (Fig. 6A). Rarely, a "puff" of dense material, probably $\mathrm{La}^{3+}$, occupied the basal lamina immediately opposite a pit or at some distance from a labeled pit or vesicle (Fig. 7).

The endothelium of cerebral arterioles in normal, untreated rats contains many pits and vesicles as in Fig. 6A (Van Deurs 1980). In segments of some arterioles and in a few capillaries (Fig. 6B), there may have been an increase in the number of these profiles after infusion of hyperosmotic arabinose.

At times, several vesicles fused to form a chain of three to five vesicles but were oriented, usually, parallel to the basal lamina rather than being directed at right angles, across the width of the vessel (Fig. 8). No chain of vesicles or other type of channel that could connect vessel lumen with perivascular basal lamina was encountered in any of the sections - single or serial. Some vesicles communicated with the intercellular cleft on either side of a tight junction. Such pits were single, occasional, and not members of a cluster (Figs. 2, 6A). Most of the filled, interjunctional pools did not communicate with a labeled pit.

The cytoplasm of a few endothelial cells was flooded with either HRP or $\mathrm{La}^{3+}$. In such vessels, the freely dispersed tracer either filled one endothelial cell while being excluded from the matrix of its neighbor, or could inundate all of the endothelial cells of a capillary segment. It is emphasized that the HRP or $\mathrm{La}^{3+}$ did not enter the basal lamina around most of the blood vessels with flooded endothelium.

\section{Discussion}

A hypertonic solution of the non-electrolyte, arabinose, opens the blood-brain barrier to ionic lanthanum by rendering the endothelial junctions permeable. The simultaneous use of both tracers permitted the selection of cerebral regions that would be likely to contain extravasated $\mathrm{La}^{3+}$ which, at low concentration, is invisible in the light microscope. However, the electrondense $\mathrm{La}^{3+}$ deposit probably obscured the route of passage taken by the larger but less dense HRP.

The passage of the $\mathrm{La}^{3+}$ was through tight junctions between endothelial cells. As with the intracarotid infusion of $3 \mathrm{M}$ urea in rabbits, the outer leaflets did not become discernibly separated (Brightman et al. 1973) but $\mathrm{La}^{3+}$ was able to penetrate successive, interjunc- tional pools. Lanthanum permanganate fixation reveals a $5 \mathrm{~nm}$-thick extracellular layer firmly bound to the plasma membrane (Lesseps 1967). Ionic lanthanum presumably infiltrates this layer. If the tight junctions had become separated during cell shrinkage, the median lamina of the pentalaminar tight junctions should have become thickened in unstained sections. No obvious thickening, i.e., penetration of $\mathrm{La}^{3+}$ into the junction occurred. The presumed thickening of the median lamina would probably be a slight one and, as the cells are very likely rehydrated during fixation (Brightman et al. 1973) only a small amount of $\mathrm{La}^{3+}$ would be trapped in the junctional cleft. The only evidence of junctional alteration was the entry of $\mathrm{La}^{3+}$ into interjunctional lacunae that are normally inaccessible to ionic lanthanum (Bouldin and Krigman 1975).

The entry of $\mathrm{La}^{3+}$ into the interjunctional lacunae of cerebral endothelium, like the entry of HRP in the rabbit during exposure to hyperosmotic urea (Brightman et al. 1973) and of colloidal lanthanum in the rat after treatment with hyperosmotic mannitol (Nagy et al. 1979), supports the hypothesis that shrinkage of the endothelial cells renders their junctions permeable (Rapoport et al. 1972; Rapoport 1978). That the colloidal lanthanum did not often penetrate every interjunctional pool appearing in a single section of rat vessels, was attributed to the three dimensional complexity of the interendothelial clefts (Nagy et al. 1979).

Lanthanum, in its ionic form, is the smallest available electron-dense tracer (mol. wt. 138.9, ionic radius $1.15 \AA$ ) (Harned and Owen 1958). Although the $\mathrm{La}^{3+}$ may be trapped by the negatively charged groups of the basal lamina and though $\mathrm{LaCl}_{3}$ was included in the buffer rinses and osmium solutions after aldehyde fixation, the lanthanum was unpredictably lost from portions of the basal lamina. The $\mathrm{La}^{3+}$ may have been removed during fixation, washing, or sectioning. Such a patchy loss has never been observed with HRP and is a disadvantage of lanthanum usage. The absence of lanthanum deposit from a portion of the basal lamina does not necessarily mean that no lanthanum had penetrated that part. A "puff" of $\mathrm{La}^{3+}$ that had been released from a fusing vesicle may be even more prone to removal during the aldehyde perfusion. Nevertheless, once the $\mathrm{La}^{3+}$ had entered abluminal pits, cytoplasmic and interjunctional pools, it was usually retained.

The exudation of probe molecule appears to take place in the cortex where arterioles penetrate the brain rather than in deeper regions where capillary plexuses may predominate. It is emphasized that usually those clefts between endothelial cells of arterioles rather than capillaries examined in serial sections were penetrated 
completely from luminal to abluminal surfaces in a single, thin section. The failure of probe molecules, such as HRP, to reach these pools in capillaries under a variety of states that lead to the extravasation of the tracer has been reasonably taken to mean that the escape across capillaries is not by way of junctions (Beggs and Waggener 1976; Horton and Hedley-Whyte 1979; Lossinsky et al. 1979; Nag et al. 1977, 1979; Persson and Hansson 1976; Petito 1979; Petito et al. 1977; Povlishock et al. 1978; Van Deurs 1976; Westergaard 1975, 1980; Westergaard et al. 1977, 1978).

An equally plausible explanation is that even very few patent clefts could account for the observed rapid escape of blood-born molecules and ions. The endothelial surface area that is opened to water-soluble molecules, such as sucrose and protein, need only be $0.001 \%$ of the total to account for their escape from blood. This fraction of the capillary surface area has been estimated (by S.R.) for sucrose as follows. Let $\mathrm{P} \mathrm{cm} \cdot \mathrm{s}^{-1}=$ cerebrovascular permeability to ${ }^{14} \mathrm{C}$ sucrose, $A=$ capillary surface area $\left(240 \mathrm{~cm}^{2} \cdot \mathrm{g}^{-1}\right.$ brain), $\mathrm{D}=$ aqueous diffusion coefficient for sucrose $\left(5 \cdot 10^{-6} \mathrm{~cm}^{2} \cdot \mathrm{s}^{-1}\right)$ and $\delta=$ diffusion path length across endothelium $\left(\sim 5 \cdot 10^{-5} \mathrm{~cm}\right)$. For diffusion through aqueous pores within the cerebrovascular endothelium, the fraction $f$ of surface area for diffusion following barrier opening is $f=\frac{(\mathrm{PA}) \delta}{\mathrm{DA}}$ where, for ${ }^{14} \mathrm{C}$-sucrose, $\mathrm{PA}=200 \cdot 10^{-6} \cdot \mathrm{s}^{-1}$ (Rapoport et al. 1980), the approximate value for altered vessels following $1.6 \mathrm{M}$ arabinose infusion. Substituting this value of PA and the values above for the other parameters in the equation, give $f=1 \cdot 10^{-5}$. Thus, only $0.001 \%$ of the endothelial surface need become accessible to ${ }^{14} \mathrm{C}$ sucrose to account for increased ${ }^{14} \mathrm{C}$-sucrose permeability following osmotic opening of the barrier. The chance of finding such rare openings in single, thin section is, therefore, small.

No structural alteration of the endothelial tight junctions was seen in freeze-fractured brain capillaries of the lizard Anolis Carolinensis when the blood-brain barrier to peroxidase was opened by hyperosmotic $D-$ glucose (Shivers 1979). The assumption, in these experiments, was that the fractured sample was that of vessels rendered permeable. However, the vessels were haphazardly affected. The HRP exudates serve as guides for selecting the regions to be fractured, and any given exudate probably encompasses both affected and unaffected blood vessels. It cannot be assumed, therefore, that the plane of fracture had gone through the tight junction of an affected vessel rather than its unaffected neighbor.

In apparent contradiction to the entry of HRP into the interjunctional pools of the rabbit's endo- thelium following its exposure to hyperosmotic urea (Brightman et al. 1973), there was no such penetration in the present experiments where only HRP had reached the endothelium. This discrepancy may be accounted for by three possibilities: the multifocal osmotic opening involves only a fraction of the vascular bed and the HRP coating the abluminal face of an endothelial cell crossed via a cleft in another region of the vessel (Brightman 1977); the junctions of affected segments of endothelium may have become permeable to both tracers but the $\mathrm{La}^{3+}$, being more electrondense, masked the HRP reaction product; thirdly, the much larger protein (HRP) molecule was transferred vesicularly across the affected endothelium whereas the smaller $\mathrm{La}^{3+}$ could pass through junctions.

The possibility that damage to the endothelium can be a mechanism of solute extravasation is unlikely (Brightman 1977; Brightman et al. 1973). If the injury is in the form of an actual tear across the endothelium, it would have to be small enough to prevent blood cells from passing, since there is no hemorrhage in the exudates. Such narrow tears would be as difficult to find as the open junctions. The endothelial cell membrane may be rendered permeable to substances as large as HRP during fixation, especially when the vessel had been perfused with hyperosmotic media, fixed and subsequently infused with the HRP (Brightman 1977). In such vessels, the HRP was usually confined to the endothelial cell cytoplasm, and only rarely entered the perivascular basal lamina, much less the neuropil.

Barrier opening has been attributed to vesicular transfer or the active formation of transendothelial channels by many authors. It is possible, but unlikely, that vesicular transport could account for the major portion of protein extravasation following hyperosmotic infusions (cf. Westergaard 1980). HRP can exude from vessels that had been exposed to hyperosmotic arabinose, then fixed and, one to several hours later, infused with HRP (Brightman 1977). Several hours of fixation should halt vesicle formation and movement. It was concluded that the HRP escaped through channels - junctional or parajunctional - that had formed during life and were fixed in the open state.

Active formation of transendothelial channels has been proposed to account for HRP extravasation under different conditions, e.g., spinal cord concussion (Beggs and Waggener 1976), cerebral ischemia (Lossinsky et al. 1979) and hyperosmotic treatment (Shivers 1979). Such continuous channels that connect blood and perivascular fluid have not been encountered in our specimens. If they did form, the estimate of the area of endothelium affected by osmotic solutions suggests that the probability of finding such parajunctional channels would be, likewise, remote. Although a few patent clefts were found in single, thin 
sections, we have yet to see a vesicle or channel span the width of the cerebral endothelium. Transcellular channels are recognizable across the endothelium of the choroid plexus (Van Deurs 1980) and across the breadth of reactive arachnoidal cells (Rosenstein and Brightman, unpubl. data). Their absence from parenchymal endothelium intimates that the properties of this membrane differ from choroidal and arachnoidal plasma membranes.

It remains possible that channels may form across the endothelium under certain, but not all, experimental conditions that lead to opening of the blood-brain barrier. However, their role in permeability of cerebral endothelium is not clear. The hagfish has a tight blood brain barrier, yet its brain capillary endothelium has both tight junctions and many cytoplasmic vesicles and tubules. The tubules and vesicles are numerous and may extend almost across the width of the endothelium but do not span its entire thickness. The tubules are in continuity with either the abluminal or luminal surfaces. These vesicles and tubules, therefore, are not involved in the transendothelial transport of macromolecules, because only a few of them were labeled following the injection of microperoxidase and the tracer did not reach the basal lamina or the brain parenchyma (Bundgaard et al. 1981). In serial reconstructions of confluent vesicles in this type of endothelium, racemose aggregates of vesicles appear to be stable, fixed structures which, though numerous, do not bring the vessel lumen into continuity with the perivascular fluid (Bundgaard et al. 1979). Thus, the presence of numerous vesicles and tubules within cerebral endothelium does not necessarily connote the transendothelial transport of macromolecules. Whether a hyperosmotic stimulus in this species leads to a very rapid creation of parajunctional channels open to both faces of an endothelium already laced by preexisting, deep invaginations of the cell membrane has yet to be ascertained. The present experiments do demonstrate clearly, however, that the blood-brain barrier to small solutes can be lowered by the opening of successive tight junctions between endothelial cells of the cerebral vasculature.

\section{References}

Beggs JL, Waggener JD (1976) Transendothelial vesicular transport of protein following compression injury to the spinal cord. Lab Invest 34:428-439

Bouldin TW, Krigman MR (1975) Differential permeability of cerebral capillary and choroid plexus to lanthanum ion. Brain Res 99:444-448

Brightman MW (1977) Morphology of blood-brain interfaces. In: Bito LZ, Davson H, Fenstermacher JD (eds) The ocular and cerebrospinal fluids. Exp Eye Res [Suppl] 25:1-25
Brightman MW, Reese TS (1969) Junctions between intimately apposed cell membranes in the vertebrate brain. J Cell Biol $40: 648-677$

Brightman MW, Hori M, Rapoport SI, Reese TS, Westergaard E (1973) Osmotic opening of tight junctions in cerebral endothelium. J Comp Neurol 152: $317-326$

Bundgaard M, Cserr H, Murray M (1981) Impermeability of Hagfish cerebral capillaries to radiolabelled polyethylene glycols and to microperoxidase. Brain Res 206:71-81

Bundgaard M, FroKjaer-Jensen J, Crone C (1979) Endothelial plasmalemmal vesicles as elements in a system of branching invaginations from the cell surface. Proc Natl Acad Sci USA $76: 6439-6442$

Castel M, Sahar A, Erlij D (1974) The movement of lanthanum across diffusion barriers in the choroid plexus of the cat. Brain Res 67:178-184

Chiueh CC, Sun CL, Kopin IJ, Fredericks WR, Rapoport SI (1978) Entry of $\left[{ }^{3} \mathrm{H}\right]$ norephinephrine, $\left[{ }^{125} \mathrm{I}\right] \mathrm{albumin}$ and Evans blue from blood into brain following unilateral osmotic opening of the blood-brain barrier. Brain Res 145:291-301

Harned HS, Owen BB (1958) The physical chemistry of electrolytic solutions 3rd edn. Reinhold, New York, pp 164, 700, 702

Horton JC, Hedley-Whyte ET (1979) Protein movement across the blood-brain barrier in hypervolemia. Brain Res 169:610-614

Lesseps RJ (1967) The removal by phospholipase C of a layer of lanthanum-staining material external to the cell membrane in embryonic chick cells. J Cell Biol 34:173-183

Lossinsky AS, Garcia JH, Iwanowski L, Lightfoote WE, Jr (1979) New ultrastructural evidence for a protein transport system in endothelial cells of gerbil brains. Acta Neuropathol (Berl) 47:105- 110

Nag S, Robertson DM, Dinsdale HB (1977) Cerebral cortical changes in acute experimental hypertension. An ultrastructural study. Lab Invest 36:150 - 161

Nag S, Robertson DM, Dinsdale HB (1979) Quantitative estimate of pinocytosis in experimental acute hypertension. Acta Neuropathol (Berl) 46:107-116

Nagy Z, Pappius HM, Mathieson G, Hüttner I (1979) Opening of tight junctions in cerebral endothelium. 1. Effect of hyperosmolar mannitol infused through the internal carotid artery. J Comp Neurol 185:569-578

Persson L, Hansson H-A (1976) Reversible blood-brain barrier dysfunction to peroxidase after a small stab wound in the rat cerebral cortex. Acta Neuropathol (Berl) 35:333-342

Petito CK (1979) Early and late mechanisms of increased vascular permeability following experimental cerebral infarction. $\mathrm{J}$ Neuropathol Exp Neurol 38:222-234

Petito CK, Schaefer JA, Plum F(1977) Ultrastructural characteristics of the brain and blood-brain barrier in experimental seizures. Brain Res 127:251-267

Povlishock JT, Beck DP, Sullivan HG, Miller JD (1978) Vascular permeability alterations to horseradish peroxidase in experimental brain injury. Brain Res 153:223-239

Rapoport SI (1978) Osmotic opening of the blood-brain barrier. In: Cerebral vascular smooth muscle and its control. Ciba Foundation Symposium. Elsevier/Excerpta Medica, Amsterdam, pp 237-255

Rapoport SI, Hori M, Klatzo I (1972) Testing of a hypothesis for osmotic opening of the blood-brain barrier. Am J Physiol $223: 323-331$

Rapoport SI, Fredericks WR, Ohno K, Pettigrew KD (1980) Quantitative aspects of reversible osmotic opening of the bloodbrain barrier. Am J Physiol 238:421-431

Shivers RR (1979) The effect of hyperglycemia on brain capillary permeability in the lizard, anolis carolinensis. A freeze-fracture analysis of blood-brain barrier pathology. Brain Res 170 . $509-522$ 
Van Deurs B (1976) Observations on the blood-brain barrier in hypertensive rats, with particular reference to phagocytic pericytes. J Ultrastruct Res 56:65-77

Van Deurs B (1980) Structural aspects of brain barriers, with special reference to the permeability of the cerebral endothelium and choroidal epithelium. Int Rev Cytol 65:117-119

Westergaard E (1975) Enhanced vesicular transport of exogenous peroxidase across cerebral vessels, induced by serotonin. Acta Neuropathol (Berl) 32:27-42

Westergaard E, Hertz MM, Bolwig TG (1978) Increased permeability to horseradish peroxidase across cerebral vessels, evoked by electrically induced seizures in the rat. Acta Neuropathol (Berl) $41: 73-80$
Westergaard E, Van Deurs B, Brondsted HE (1977) Increased vesicular transfer of Horseradish peroxidase across cerebral endothelium, evoked by acute hypertension. Acta Neuropathol (Berl) $37: 141-152$

Westergaard E (1980) Ultrastructural permeability properties of cerebral microvasculature under normal and experimental conditions after application of tracers. In: Cervos-Navarro J, Ferszt $\mathrm{R}$ (eds) Advances in neurology, vol 28. Brain edema. Raven Press, New York, pp 55-74

Received December 13, 1982/Accepted January 17, 1983 